\title{
Tumor phyllodes maligno recurrente, reporte de un caso con comportamiento agresivo, importancia del tratamiento multidisciplinario
}

\author{
Recurrent malignant phyllodes tumor, a case report with \\ aggressive behavior, importance of multidisciplinary treatment \\ Luis Israel Llerena Béjar, ${ }^{*}$ Arturo Pabel Miranda Aguirre, ${ }^{*}$ Marytere Herrera Martínez, \\ Daniel Garza, ${ }^{*}$ Eva Ruvalcaba Limón, ${ }^{*}$ Carlos Domínguez, ${ }^{*}$ Juan Alberto Tenorio Torres, ${ }^{*}$ \\ Felipe Villegas Carlos, ${ }^{*}$ Guillermo Moreno Flores*
}

* Médico Ginecólogo, Alta Especialidad en Mama del Instituto de Enfermedades de la Mama-FUCAM. Ciudad de México, México.
Correspondencia: Dr. Luis Israel Llerena Béjar E-mail: israelllerena@ hotmail.es

\section{RESUMEN}

El tumor phyllodes maligno es uno de los tumores fibroepiteliales que representa el $1 \%$, caracterizado por el crecimiento excesivo del estroma y aumento de mitosis, con recurrencias locales del 23 al $30 \%$ dentro de los dos a tres años, el tratamiento es la escisión quirúrgica con márgenes mayores a $1 \mathrm{~cm}$ para evitar las recurrencias, seguida de radioterapia, sin evidencia de quimioterapia. Se presenta el caso de una mujer de edad adulta, con tumor phyllodes maligno, con múltiples recurrencias, que tiene un comportamiento agresivo, con tratamiento multimodal, tratada con cirugía, radioterapia y quimioterapia.

Palabras clave: Tumor phyllodes, tumor de mama.

\section{ABSTRACT}

The malignant phyllodes tumor is one of the fibroepithelial tumors that represents $1 \%$, symptoms due to excessive stromal growth, increased mitosis, with local recurrences of 23 to $30 \%$ within 2 to 3 years, the treatment is excision surgical with margins greater than $1 \mathrm{~cm}$ to avoid recurrences, quickly of radiotherapy, without evidence of chemotherapy. We present the case of an adult woman, with a malignant phyllodes tumor, with multiple recurrences, who has an aggressive behavior, with multimodal treatment, treated with surgery, radiotherapy and chemotherapy.

Keywords: Malignant phyllodes, breast tumor.

Citar como: Llerena BLI, Miranda AAP, Herrera MM, Garza D, Ruvalcaba LE, Domínguez C et al. Tumor phyllodes maligno recurrente, reporte de un caso con comportamiento agresivo, importancia del tratamiento multidisciplinario. Rev Mex Mastol. 2020; 10 (1): 28-30. https://dx.doi.org/10.35366/95765 


\section{INTRODUCCIÓN}

El tumor phyllodes es un tumor bifásico fibroepitelial, de comportamiento biológico distinto, representa el 0.3 al $1 \%$ de todos los tumores de la mama, 2-3\% de los tumores fibroepiteliales, el tumor phyllodes maligno muestra una combinación de marcado pleomorfismo nuclear de las células estromales, un crecimiento excesivo del estroma definido como la ausencia de elementos epiteliales, mitosis incrementadas (mayor o igual a 10 por 10 campos de gran aumento [HPF]), estroma incrementado, la celularidad que suele ser difusa y las fronteras infiltrativas.

Las recurrencias locales pueden ocurrir en el 23 a 30\% en tumores phyllodes malignos, generalmente se desarrollan dentro de dos a tres años. Un margen quirúrgico afectado es el único factor asociado con un mayor riesgo de recurrencias locales (cociente de riesgo [HR] 4.673).

El objetivo del estudio es presentar un caso clínico de un tumor phyllodes maligno recurrente, de comportamiento agresivo con tratamiento multimodal.

\section{PRESENTACIÓN DEL CASO}

Mujer de 60 años, con diagnóstico de tumor phyllodes de crecimiento rápido, tratada con mastectomía total + reconstrucción con rota- ción de colgajo dorsal ancho, y un segundo tiempo de remodelación y avance de colgajos, con reporte de tumor phyllodes maligno de 35 $\mathrm{cm}$, sobre crecimiento estromal en el 100\%, necrosis $10 \%$, bordes 0.5 el más cercano (lecho quirúrgico), presentando a los cinco meses recurrencia a nivel de cicatriz y colgajo inferior, en febrero 2018 escisión amplia y radioterapia en parrilla costal $60 \mathrm{GY}$. Al año de la primera cirugía, nueva recaída en región dorsal sobre cicatriz quirúrgica y en sitio de emergencia de drenaje (agosto 2018 escisión amplia), nueva progresión en diciembre del 2018 en hipocondrio derecho y sitio de cicatriz quirúrgica (Figura 1), en enero 2019 resección amplia de zona abdominocostal en bloque y resección de lesión satélite y cobertura cutánea con VRAM (Figura 2), durante el postoperatorio se documentan cutánides y es llevada a resección de las mismas, en febrero recibió un ciclo de adriamicina, actualmente cursando su cuarto ciclo de ocho de gemcitabina/docetaxel, con respuesta parcial.

\section{DISCUSIÓN}

La mayor parte de las recidivas locales se producen en la localización de la resección inicial, la radioterapia selectiva puede ser tan eficaz en términos de reducción de recurrencias como la irradiación de la totalidad de la mama y se presenta en los dos primeros años como es el caso que presentamos.

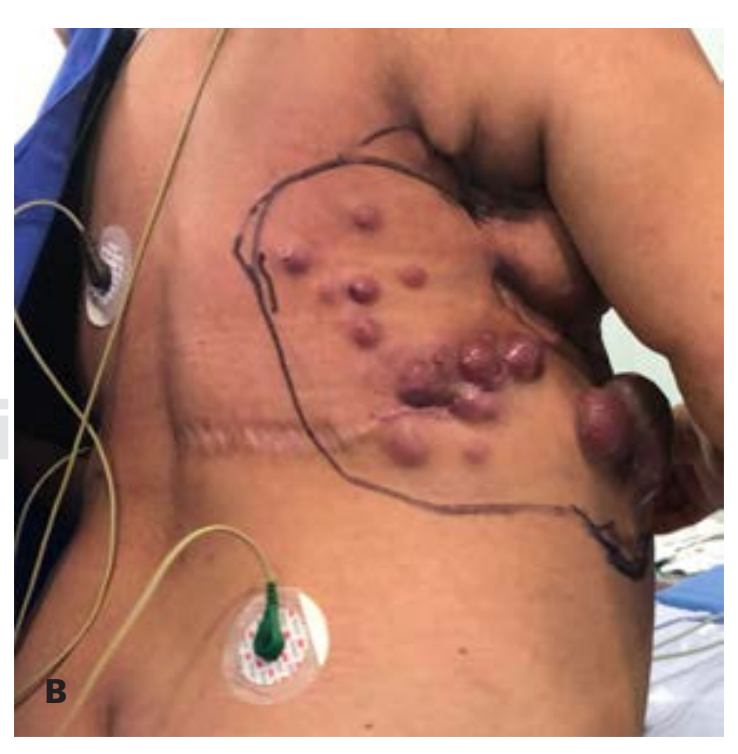

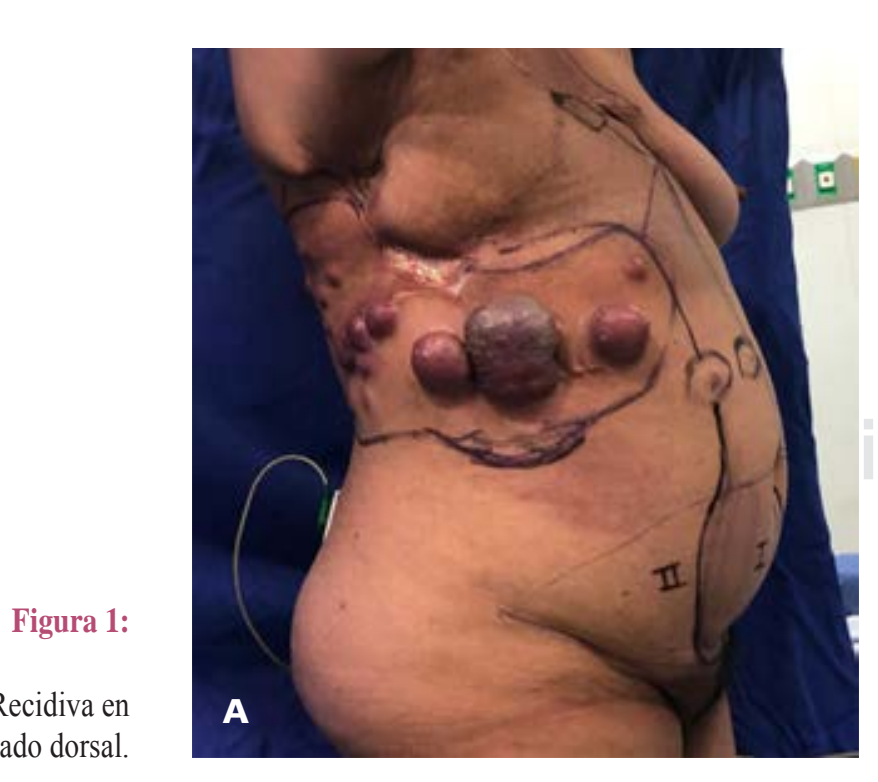


Figura 2:

A y B) Cobertura cutánea VRAM.

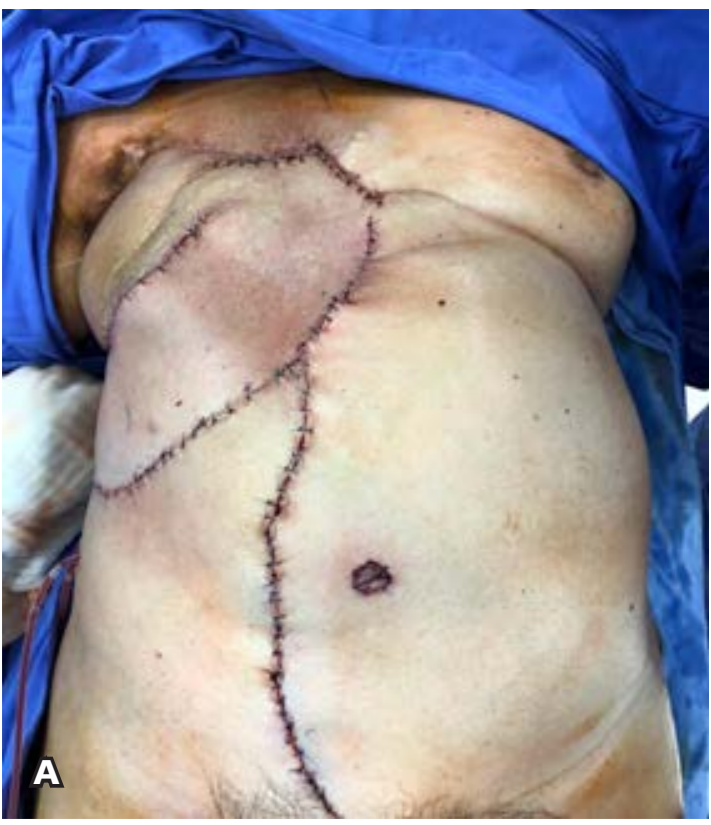

El principal factor de impacto sobre la recidiva tumoral es la presencia de márgenes quirúrgicos de resección afectos. El porcentaje de recidiva varía desde un $10-17 \%$ en las formas benignas, hasta el $40 \%$ en los subtipos malignos.

El tratamiento está encaminado con cirugía y radioterapia en cirugía conservadora con tamaños $>2 \mathrm{~cm}$ o margen $<1 \mathrm{~cm}$ o positivo sin posibilidad de rescisión, tamaños $>5 \mathrm{~cm}$ o ganglios positivos. Hay una limitación en la información en la literatura para el uso de quimioterapia; sin embargo, se describe como tratamiento paliativo ifosfamida + /- doxorrubicina o cisplatino, ya que prolonga la supervivencia y retrasa el progreso de metástasis.

\section{CONCLUSIONES}

El tratamiento se basa en la cirugía, intentado obtener márgenes de resección libres de enfermedad, asociado con radioterapia adyuvante, destacamos la importancia del diagnóstico

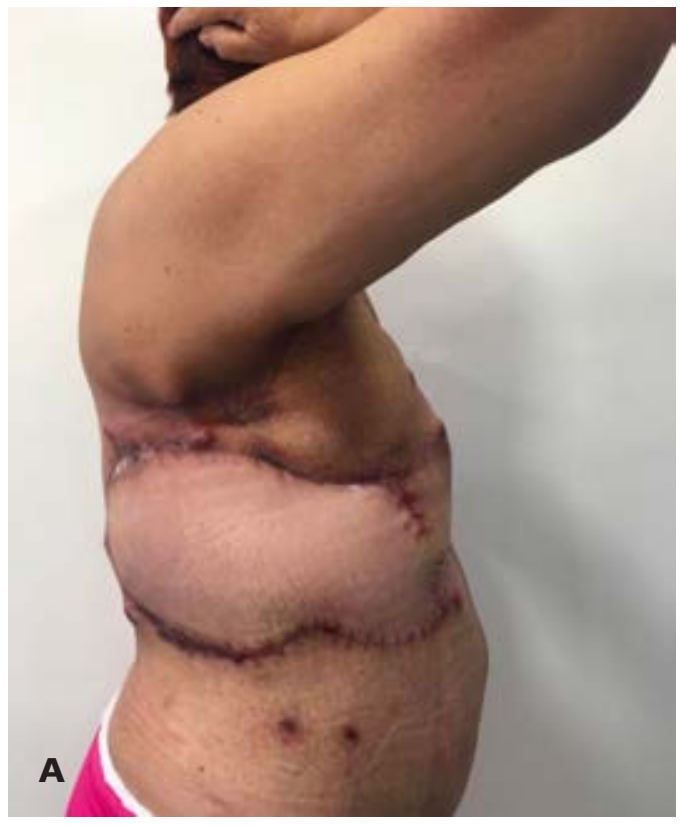

precoz y de la educación de las mujeres y sobre todo el tratamiento multidisciplinar con el equipo de Cirugía Oncológica, Cirugía Plástica, Radioterapia y Oncología Médica.

\section{LECTURAS RECOMENDADAS}

1. Li J, Tsang JY, Chen $\mathrm{C}$ et al. Predicting outcome in mammary phyllodes tumors: relevance of clinicopathological features. Ann Surg Oncol. 2019; 26 (9): 2747-2758. doi: 10.1245/s10434-019-07445-1.

2. Limaiem F, Waheed A. Cáncer, phyllodes tumor de la mama (cistosarcoma) [Actualizado en 2019 el 4 de junio]. En: StatPearls [Internet]. Treasure Island (FL): StatPearls Publishing; 2019. Disponible en: https:// www.ncbi.nlm.nih.gov/books/NBK541138/

3. Chao X, Chen K, Zeng J et al. Adjuvant radiotherapy and chemotherapy for patients with breast phyllodes tumors: a systematic review and meta-analysis. BMC Cancer. 2019; 19 (1): 372. doi: 10.1186/s12885-0195585-5.

4. Tan BY, Acs G, Apple SK et al. Phyllodes tumours of the breast: a consensus review. Histopathology. 2016; 68 (1): 5-21. doi: 10.1111/his.12876.

5. Jiménez-López J, Márquez-Acosta G, Olaya Guzmán EJ, Gómez-Pue D, Arteaga Gómez AC. Tumor phyllodes. Perinatol Reprod Hum. 2013; 27 (2): 106-112. 